\title{
Pain and Wound Healing in Surgical Patients
}

\author{
Lynanne McGuire, Ph.D. \\ University of Maryland Baltimore County \\ Kathi Heffner, Ph.D. \\ Ohio University
}

\author{
Ronald Glaser, Ph.D., Bradley Needleman, M.D., William Malarkey, M.D., Stephanie Dickinson, Ph.D., \\ Stanley Lemeshow, Ph.D., Charles Cook, M.D., Peter Muscarella, M.D., William Scott Melvin, M.D., \\ Edwin Christopher Ellison, M.D., and Janice K. Kiecolt-Glaser, Ph.D. \\ The Ohio State University
}

\begin{abstract}
Background: Human and animal laboratory studies have shown that stress delays healing of standardized punch biopsy wounds. Purpose: This 5-week prospective study of 17 women who underwent elective gastric bypass surgery addressed the association between postsurgical pain intensity and subsequent healing of a standard 2.0-mm punch biopsy wound. Methods: Participants were assessed 1 week before surgery, within $3 \mathrm{hr}$ before surgery, 1 to 3 days postsurgery, and at weekly intervals for 4 weeks following surgery. Results: Patient ratings of greater acute postsurgical pain, averaged over Days 1 and 2 postsurgery, and greater persistent postsurgical pain, averaged over 4 weekly postsurgery pain ratings, were significantly associated with subsequent delayed healing of the punch biopsy wound. Presence of depressive symptoms on the day of surgery, pre-existing persistent pain, and medical complications following initial discharge from the hospital were not related to wound healing. Depressive symptoms on the day of surgery and pre-existing persistent pain did predict persistent postsurgical pain intensity. Conclusions: These findings extend the previous laboratory models of wound healing to a surgical population, providing the first evidence that pain plays an important role in postsurgery wound healing, a key variable in postsurgical recovery.
\end{abstract}

(Ann Behav Med 2006, 31(2):165-172)

\section{INTRODUCTION}

Pain contributes to poor quality of life, reduced well-being, physical disability, and mortality $(1,2)$. One source of pain

This research was supported in part by National Institutes of Health (NIH) grants P50 DE13749, PO1 AG16321, and R37 MH42096; by NIH General Clinical Research Center Grant MO1-RR- 0034; and by Comprehensive Cancer Center Core Grant CA16058. We gratefully acknowledge the assistance of Laura von Hoene in data collection and the staff of the General Clinical Research Center for completing the punch biopsy standard wounds. We also thank Dr. Jennifer Haythornthwaite and Dr. Robert Edwards for their comments on a previous draft of this article.

Reprint Address: L. McGuire, Ph.D., Psychology Department, University of Maryland Baltimore County, 1000 Hilltop Circle, Baltimore, MD 21250. E-mail: lmcguire@umbc.edu

(C) 2006 by The Society of Behavioral Medicine. includes surgical procedures. Unrelieved postsurgical pain is common and may be a useful context in which to study pain-related morbidity and mortality. For example, postoperative pain in hip fracture patients predicted longer hospital length of stay (LOS), delayed ambulation, and long-term functional impairment (3). Attempts have been made to resolve the pervasive problem of undertreated acute postsurgical pain through development and implementation of quality improvement guidelines (4). Despite these efforts, unrelieved postsurgical pain remains an important clinical problem $(5,6)$ and may have serious implications for the recovery of surgical patients (7). The question of interest for this study was whether postsurgical pain is associated with delayed wound healing.

Why might pain influence wound healing? Pain-wound healing links can be considered within the broader context of pain-associated neuroendocrine and immune function alterations relevant to the wound repair process. Wound healing processes involve interactions of a wide array of soluble mediators and cells, and the immune system plays a key role in the early wound repair cascade $(8,9)$. Consistent overlap between painrelated dysregulation of neuroendocrine and immune function (10-13) and the aspects of neuroendocrine and immune function shown to be critical in wound healing (14-19) suggests that pain may play a key role in delayed wound healing.

In this study, we assessed the relationship between postsurgical pain and healing of a standard punch biopsy wound in gastric bypass patients. We hypothesized that patients who reported surgery-related higher intensity pain during 4 weeks postsurgery would show subsequent delayed healing of the standard wound. Because depression has been shown to influence patients' experience of pain severity (20), and has been associated with delayed healing of chronic lower leg wounds (21), we also assessed the relationship between depressive symptoms and healing of the standard wound. Additionally, we assessed the association between pre-existing persistent pain and wound healing to control for potential presurgery pain-related changes in neuroendocrine and immune function that could influence postsurgery wound healing.

\section{METHOD}

\section{Participants}

The participants were 17 patients who underwent elective gastric bypass surgery. Exclusion criteria included health prob- 
lems (other than obesity) with an immunological or endocrinological component (e.g., cancer, diabetes) and coincident surgery scheduled to occur with the gastric bypass procedure. All patients were female, aged 21 to 48 years $(M=37.65, S D=$ 7.79). The majority of patients $(65 \%)$ were Caucasian, $29 \%$ were African American, and 6\% were another minority. Most participants were married $(65 \%)$ or living with a partner $(17 \%)$ and were currently employed (82\%). All patients had completed high school; $59 \%$ had completed some college, $12 \%$ graduated college, and $12 \%$ had completed graduate or professional training. Total annual income was greater than $\$ 50,000$ for $65 \%$ of participants.

\section{Procedure}

Patients were recruited from a medical clinic specializing in gastric bypass surgery and were approached following successful completion of an initial appointment that determined their eligibility for the surgery. All patients received the same surgical procedure, roux-en-Y gastric bypass. Four patients underwent conventional surgery, which requires one midline incision, and 13 patients received minimally invasive laparoscopic surgery. Patients received a standard range of narcotic and non-narcotic analgesics for control of postsurgery pain, including intravenous morphine by patient-controlled pump, nonsteroidal anti-inflammatory agents, and acetaminophen. Informed consent was obtained, and participants were assessed 1 week before surgery, within $3 \mathrm{hr}$ before surgery, 1 to 3 days postsurgery, and at weekly intervals for 4 weeks following surgery. The protocol was approved by the Institutional Review Board at The Ohio State University.

\section{Measures}

Postsurgery pain. Pain was assessed using a 10-point numerical rating scale from 1 (no pain) to 10 (intense pain); similar numerical rating scales have been widely used for pain measurement and have demonstrated reliability and validity for assessing pain intensity (22). Participants rated their level of pain on Days 1 and 2 postsurgery and their average level of pain over the past week at weekly intervals through 4 weeks postsurgery. Acute postsurgical pain is the average of the respondents' pain ratings from the first 2 days postsurgery. Persistent postsurgical pain is the average of participants' weekly pain ratings through 4 weeks postsurgery.

Pre-existing pain. A single item from the Short Form-36 (23), "How much bodily pain have you had during the past 4 weeks?" was used to assess pre-existing pain at 1 week before surgery; pain ratings ranged from 1 (none) to 6 (very severe).

Depressive symptoms. Depressive symptoms were assessed using the Beck Depression Inventory short form (BDI-SF) (24) on the day of surgery. The BDI-SF is a 13-item measure that provides information on the severity of affective and cognitive depressive symptoms over the past week. It correlates highly with the total score of the 21 -item BDI $(r=.96)$ and with clinicians' ratings of depression $(r=.61)(24)$ and has been shown to have acceptable internal reliability and criterion group validity (25). A cutoff score of 5 has been used to indicate the threshold for clinically relevant depressive symptoms in adults $(26,27)$.

Health behaviors. Poor health behaviors (e.g., smoking) may be important risk factors for postsurgical recovery and wound healing (28). Assessment of health-related behaviors occurred at 1 week before surgery and included current use of tobacco products, caffeine intake, number of drinks in the past week, recent weight change, and sleep (29). Sleep was also assessed during hospitalization and weekly postsurgery.

Standard wound. On the day of surgery a 2-mm punch biopsy, full thickness wound was placed on the back of one upper arm of each patient prior to surgery. The area was anesthetized with $1 \%$ lidocaine with epinephrine. The punch biopsy was then performed, and bleeding was stopped using a cotton swab dipped in Monsel's solution. Antibiotic ointment was applied to the wound and the area was covered with an adhesive bandage. The punch biopsy wound provided an easily accessible, uniform shape, and standard size wound for assessment of postsurgery wound healing across patients. Digital photography was used to assess healing of the standard wound at Days 1, 2, 7, $10,14,17,21,24$, and 28 postsurgery. Included in each digital wound photograph was an "anchor"—a 2-mm black dot. A software program (30) was used to measure the area of the standard wound and the area of the anchor, and a ratio was created. Two independent raters calculated each ratio. Reliability between the independent raters was calculated using average measure intraclass correlation coefficients (ICCs). All ICCs for the standard wound measurements were above .80 except for the Day 10 measurement $(\mathrm{ICC}=.51)$. The corresponding measurements of the raters were averaged and used in the current analyses. Over time, a reduction in the size of the ratio (i.e., wound size compared to the size of the anchor) showed healing of the standard wound. A ratio of .10 indicated when the wound had healed $90 \%$.

Surgery-related outcome measures. Two primary measures were collected to indicate surgery-related complications. LOS included the number of days from the day of surgery through initial hospital discharge. One patient had a 10-day LOS in the hospital, and the remaining patients had an average LOS of 3 days $(M=3.07, S E M=0.18$, range $=2-5$ days $)$. Postsurgery complications were indicated by emergency room visits or rehospitalizations (referred to as medical contact) following initial hospital discharge. Consistent with previously reported post operative complication rates in this type of surgery (e.g., 31), $41 \%$ of patients $(n=7)$ reported emergency room contact, and $71 \%$ of those emergency room visits $(n=5)$ resulted in rehospitalization. Patient reports of medical contact were verified by hospital records. Additionally, two secondary measures were collected to indicate surgical stress: (a) duration of the surgery and (b) estimated intraoperative blood loss.

\section{Statistical Methods}

Survival analysis (32) was used to test for the relationship between acute and persistent postsurgical pain intensity and 
subsequent time to healing of the standard wound. Wound healing is the "event" and is defined as the first time point that the wound-to-anchor ratio is less than .10 and remains below .10 ( $90 \%$ healing). Censoring was used for patients with a ratio greater than .10 at the last observed time point, either followed by missing data or at Day 28. For both acute (Days 1 and 2 postsurgery) and persistent (average over the 4 weeks postsurgery) pain, two distinct pain groups were created. The acute pain analyses compared patients who reported pain intensity of less than or equal to 8 with patients who reported pain intensity greater than 8 . This level of pain intensity was chosen because it was the median split of the pain ratings $(M d n=8)$ and it provided a high-intensity acute pain comparison group (pain ratings of 9 or 10). Because several patients reported an 8 for their acute pain, the low acute pain group is larger than the high acute pain group. The persistent pain analyses also used a median split of the pain ratings and compared respondents who reported pain intensity of less than or equal to 4 with patients who reported pain intensity greater than 4 . A chi-square analysis examined the extent of overlap of the same surgical patients in the high acute pain group versus the high persistent pain group and found no significant overlap in group membership $(p>.85)$. The survival curves for time to healing were compared between the groups experiencing more or less pain using Kaplan-Meier survival estimates and the log-rank test. The log-rank test was used to test the equality of the two survivor functions, assuming proportional hazards across the groups. Because the effect of pain on healing may be confounded with factors of the surgery itself, further analyses were performed to model the effects of type of surgery, LOS, presence of postsurgery medical contact, duration of surgery, and estimated intraoperative blood loss on time to healing. The effect of depressive symptoms and pre-existing persistent pain on time to healing was also examined. Small sample sizes prevented more complicated analyses, such as Cox proportional hazards models. Missing data occurred at varying time points across participants because of technical difficulties in digital photography and scheduling of appointments. Where necessary, time to healing was calculated using Last Observation Carried Forward. Linear regressions were used to test for the association between depression at the time of surgery and postsurgery acute and persistent pain, and pre-existing persistent pain and postsurgery acute and persistent pain. Chi-square analyses were used for comparisons of health behaviors by pain groups. We used $t$ tests for comparison of mean pain between surgery types. Fisher's Exact Test was used to test for associations between LOS and pain (both dichotomized), because of small cell counts. All tests were performed with a two-sided, $\alpha=$ .05 level of significance. Stata 8.0 (33) was used for all analyses.

\section{RESULTS}

\section{Health Behaviors, Depressive Symptoms, and Pre-Existing Persistent Pain}

Health behaviors were not significantly associated with healing of the standard wound or surgery-related outcomes. There was no association between smoking and time to healing of the standard wound. The majority of patients (76\%) did not smoke, and of the 4 participants who did smoke, 2 showed relatively early healing (Day 17), and 2 showed late healing (Day 28 and after Day 28).

Survival analysis was used to examine depressive symptoms on the day of surgery and time to healing of the standard wound. The depressive symptoms groups were dichotomized as $<5$ (no depressive symptoms, $n=6$ ) or $\geq 5$ (mild or greater depressive symptoms, $n=7$ ) using the BDI-SF. The Kaplan-Meier survival curves of time to healing (90\% woundto-anchor ratio) for the two depressive symptoms groups were not significantly different (log-rank test, $p=.54$ ). Depressive symptoms did not influence healing of the standard wound. Linear regression was used to test for the relationship between depressive symptoms on the day of surgery and acute (averaged over Days 1 and 2 postsurgery) and persistent (averaged over 4 weeks postsurgery) pain. Depressive symptoms were not significantly associated with acute pain, $F(1,11)=2.12, p=.17, r=$ .4 , but did predict persistent pain, $F(1,11)=9.69, p=.01$, $r=.68$.

Survival analysis was used to examine pre-existing (previous 4 weeks) persistent pain, assessed 1 week before surgery, and time to healing of the standard wound. The pre-existing pain groups were dichotomized as $<4$ (no or low pre-existing pain, $n$ $=7$ ) or $\geq 4$ (high pre-existing pain, $n=10$ ) using the median split of the bodily pain item from the Short Form-36. The Kaplan-Meier survival curves of time to healing (90\% wound-to-anchor ratio) for the two pre-existing pain groups were not significantly different $(\log$-rank test, $p=.30)$. Pre-existing pain did not influence healing of the standard wound. Linear regression was used to test for the relationship between pre-existing pain and acute and persistent postsurgery pain. Pre-existing pain was not associated with acute pain, $F(1,15)=$ $1.01, p=.33, r=.25$, but did predict persistent pain, $F(1,15)=$ $16.20, p=.001, r=.72$.

\section{Postsurgery Pain and Wound Healing}

The first survival analysis examined acute pain and time to healing of the standard wound. The average of patients' pain ratings for the first 2 days postsurgery was used to create two pain groups. The pain rating groups were dichotomized, using the median split of the pain ratings, as $\leq 8$ or $>8$ ( $n$ s $=11$ and 6 , respectively) level of pain. Figure 1 shows the Kaplan-Meier survival curves of time to healing ( $90 \%$ wound-to-anchor ratio) for the two pain groups. Annotations on the curves indicate the number of censored observations at each time point. The group that reported less acute pain ( $\leq 8$ pain rating) had faster healing (shorter survival time). The survival curves for more or less acute pain were significantly different (log-rank test, $p=.023$ ). Patients who rated their pain lower over the first 2 days postsurgery $(\leq 8)$ experienced faster healing compared with patients who reported more acute pain postsurgery ( $>8$; e.g., median time to healing was 21 days and 28 days, respectively).

The second survival analysis examined persistent pain and time to healing of the standard wound. The average of the weekly pain ratings through 4 weeks postsurgery (1/week) was used to create two groups. The pain ratings were split at the me- 


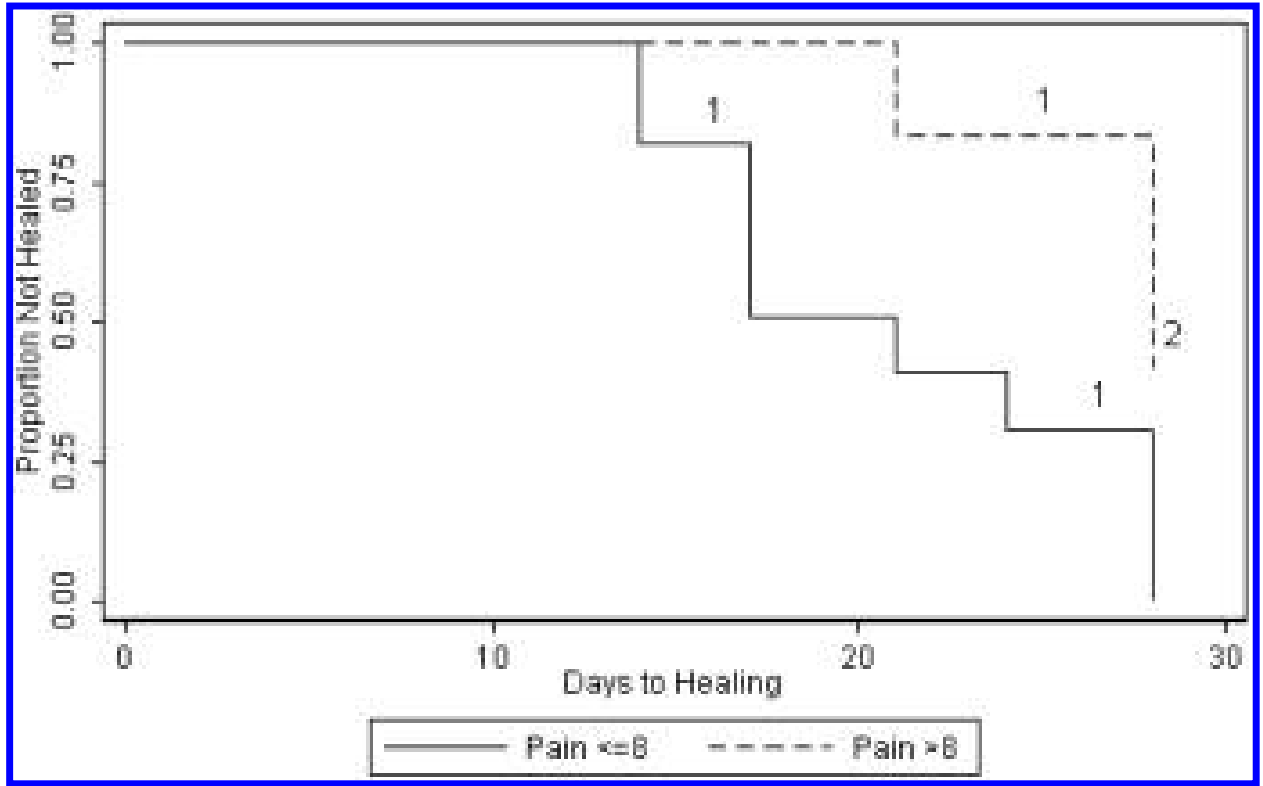

FIGURE 1 Kaplan-Meier survival curves for more or less acute pain during the first 2 days postsurgery and time to healing of the standard wound. Annotations on the curve show the number of censored observations at each time point, indicating patients who were unhealed on their last day of observation. Patients who reported higher acute pain during the first 2 days postsurgery experienced slower healing (log rank test, $p=.023)$ of the standard wound; median time to healing was 28 days.

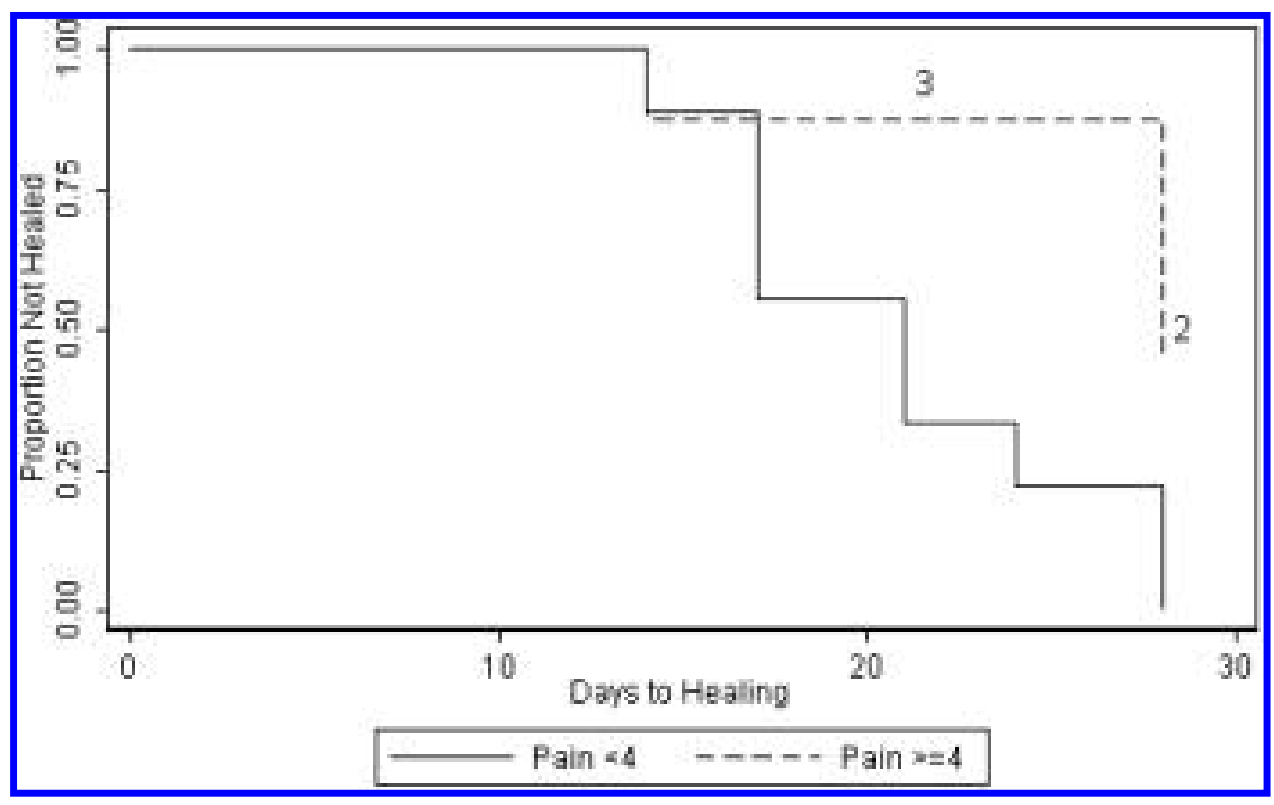

FIGURE 2 Kaplan-Meier survival curves for more or less persistent pain through 4 weeks postsurgery and time to healing of the standard wound. Annotations on the curve show the number of censored observations at each time point, indicating patients who were unhealed on their last day of observation. Patients who reported higher persistent pain during the 4 weeks postsurgery experienced slower healing (log rank test, $p=.012$ ) of the standard wound; median time to healing was 28 days.

dian, $<4$ or $\geq 4$ ( $n$ s $=8$ and 9 , respectively), to create two groups expressing more or less pain.

Figure 2 displays Kaplan-Meier survival curves of time to healing (90\% wound-to-anchor ratio) for the two pain groups. The survival curve dropped more quickly for the lower pain group $(<4)$, indicating that patients who rated their average pain lower across the 4 weeks postsurgery experienced faster healing than patients who reported higher pain $(\geq 4$; e.g., median time to healing was 21 days and 28 days, respectively). The difference in healing times between the more and less pain groups was significant (log-rank test, $p=.012$ ).

\section{Surgery-Related Outcome Measures}

To examine the possibility that the effect of pain on healing of the standard wound was confounded with factors of the surgery itself, such as type of surgery, three additional survival 
analyses were performed. Although multivariable Cox proportional hazards models are ideal for assessing confounders and effect modifiers for survival analysis, our small sample size limited our analyses to univariable comparisons of survival curves for each of the factors in question. The first analysis examined surgery type-conventional $(n=4)$ versus laparoscopic $(n=$ 13); pain; and time to healing. Although no statistically significant differences were found, laparoscopic patients reported slightly lower acute $(M=7.58, S D=1.38)$ and persistent $(M=$ $3.9, S D=1.54)$ pain compared with conventional surgery patients (acute, $M=7.75, S D=1.85$; persistent, $M=4.33, S D=$ 2.0). The difference in healing times between groups was not significant (log-rank test, $p=.42$ ); however it did appear that patients who received the laparoscopic surgery healed faster $(M d n$ $=24$ days) than conventional surgery patients ( $M d n=28$ days). To control for the effects of surgery type, the analyses examining acute and persistent pain and time to healing were repeated including only laparoscopic surgery patients. Both lower acute pain and lower persistent pain were associated with faster healing (log-rank test, $p=.017$ and $p=.035$, respectively) in the laparoscopic surgery patients.

The second survival analysis examined LOS and time to healing of the standard wound. LOS was dichotomized into $\leq 3$ days or $>3$ days ( $n s=13$ and 3 , respectively). The difference in healing times between the shorter and longer LOS groups was marginally significant (log-rank test, $p=.085$ ), suggesting that the shorter LOS group may have experienced faster healing. The relationship between pain and LOS also was examined. Acute pain was associated with LOS. All 11 of the patients who reported less pain $(\leq 8)$ in the first 2 days postsurgery spent fewer days in the hospital $(\leq 3)$; whereas, only 2 of the 5 patients reporting more pain $(>8)$ had a shorter LOS (Fisher's exact test, $p=.018$ ). Persistent pain was not related to LOS (Fisher's exact test, $p=1.0$ ). Because LOS is a confounder in the interpretation of pain and time to healing, the survival analyses examining acute and persistent pain and time to healing of the standard wound were repeated including only participants with LOS less than or equal to 3 days $(N=13)$. Although the results for acute pain and time to healing were no longer significant (log-rank test, $p=.24$ ), the central findings for greater intensity persistent pain over the 4 weeks postsurgery and delayed time to healing remained significant (log-rank test, $p=.015$; see Figure 3 ).

The third survival analysis examined medical contact after initial discharge from the hospital and time to healing of the standard wound. Medical contact was dichotomized into yes or no ( $n s=7$ and 9, respectively). The difference in healing times between medical contact groups was not significant (log-rank test, $p=.40$ ). Similarly, additional analyses examined duration of surgery and estimated intraoperative blood loss, two indicators of intraoperative stress, and found no significant associations with time to healing of the standard wound (log-rank test, $p$ $=.34$ and $p=.12$, respectively). Although not significant, both duration of surgery and intraoperative blood loss showed stronger associations with acute postsurgery pain $(r=.33, p=.19$, and $r=.29, p=.25$, respectively), compared with persistent postsurgery pain $(r=.21, p=.42$, and $r=.08, p=.75$, respectively). Estimated intraoperative blood loss was significantly associated with type of surgery. Blood loss was significantly lower in laparoscopic surgery patients compared with conventional surgery patients, $t(15)=4.34, p=.001$.

\section{DISCUSSION}

Pain is a frequent consequence of wounds, and it may have important implications for the subsequent wound healing pro-

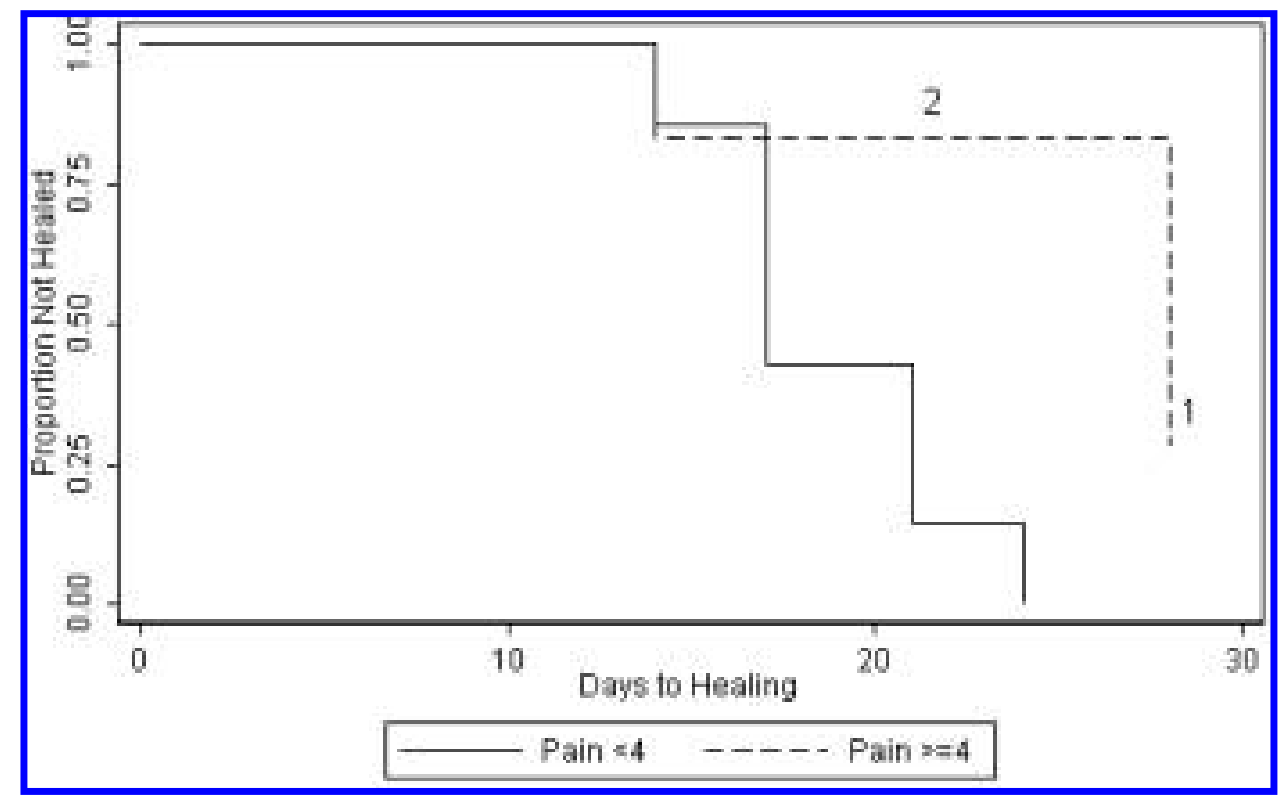

FIGURE 3 Kaplan-Meier survival curves for more or less persistent pain through 4 weeks postsurgery and time to healing of the standard wound, including only participants with a hospital stay of 3 or fewer days $(N=13)$. Annotations on the curve show the number of censored observations at each time point, indicating patients who were unhealed on their last day of observation. Patients who reported higher persistent pain during the 4 weeks postsurgery experienced slower healing (log rank test, $p=.015$ ), of the standard wound. 
cess. Pain-related delay in wound healing is particularly relevant in surgical patients, because healing of surgical wounds is a key outcome, and undertreatment of postsurgical pain is frequent $(5,6)$. In this study of female gastric bypass patients, postsurgical pain intensity was associated with subsequent delayed healing of a standard wound. For both the acute (average pain during Days 1 and 2 postsurgery) and persistent (average pain through 4 weeks postsurgery) measures of pain, higher ratings of postsurgery pain were associated with slower healing of the standard wound.

A series of analyses were performed to test for potential alternative explanations for the relationship between postsurgical pain and delayed wound healing. Although these analyses inflated the possibility of finding significant results where null findings were desirable, we found no evidence that the relationships between pain and wound healing were simply a function of health habits, depressive symptoms, pre-existing pain, or surgery-related factors. Although cigarette smoking has been linked to poorer wound healing in previous studies (34), smoking was not related to wound healing in this study. The majority of patients were nonsmokers, and it is likely that the number of smokers $(n=4)$ was too small to see the effects of smoking on wound healing. Among our patients who smoked, 2 healed relatively quickly, and 2 healed slowly. Depressive symptoms on the day of surgery were associated with higher ratings of persistent pain postsurgery but were not significantly related to acute pain postsurgery or healing of the standard wound. Pre-existing persistent pain during the month before surgery was a significant predictor of persistent postsurgical pain, but not acute pain or wound healing. Although type of surgery was not significantly related to pain or healing of the standard wound, patients who received laparoscopic surgery reported slightly lower pain and experienced faster healing ( $M d n=24$ days vs. 28 days) compared with patients who received conventional surgery. The relationships between acute and persistent pain and time to healing were maintained after controlling for type of surgery by including only patients who received laparoscopic surgery in the analyses. Presence of medical complications following initial discharge from the hospital and intraoperative indicators of surgical stress (i.e., surgery duration, blood loss) were not related to wound healing.

The relationships among postsurgery pain, LOS, and healing are more complicated. Higher acute pain in Days 1 and 2 postsurgery, but not persistent pain over 4 weeks postsurgery, was associated with longer LOS. After controlling for LOS by including only short-LOS participants in the analyses, the relationship between persistent pain and time to healing was maintained. The association between acute pain and time to healing was no longer significant. Pain in Days 1 and 2 postsurgery may also predict delayed healing, but the delayed healing may depend in part on other surgery-related factors that lead to both acute pain and a longer LOS. Alternatively, acute pain may predict both LOS and healing and may mediate the association between longer LOS and delayed healing. Most important, however, is that the pattern of results suggests that persistent post- surgery pain is an important predictor of time to healing, independent of surgery-related complications.

We chose to use a standard wound, a 2-mm full thickness punch biopsy on the arm, rather than the patients' surgical wounds. The use of the punch biopsy wound provided us with a standard size, depth, and shape wound that we could compare across participants. Actual surgical wounds differed in size and shape across patients and, more important, the standard procedure of suturing the wound closed and then covering the closed surgical wounds with adhesive wound closure strips meant that there was not an open wound that could be measured, a barrier to wound assessment for virtually all kinds of surgeries.

Our findings suggest a need for systematic, prospective examination of pain specifically related to the progress of wound healing and evaluation of possible underlying mechanisms, including pain-related neuroendocrine and immune function alterations relevant to wound healing. In the case of surgery patients, the prospective evaluation of pain and wound healing need not wait until postsurgery. There is some evidence to suggest that presurgical pain tolerance, as measured by the cold pressor task, predicts postsurgical immune function and complications (35). Given the current findings, a question of interest is whether presurgical pain tolerance predicts who is at risk for delayed postsurgery wound healing. Pain-related poor outcomes in wound healing could have important implications for patient morbidity, including an increased risk of infection (36) and delayed return to independent functioning. These relationships may be even more critical in older adults or persons with comorbid systemic illness, such as diabetes, as age- or disease-related immune dysregulation may already present a challenge for successful wound healing (37). This study included young to middle-age women, and thus the extent to which the findings of this study generalize to men and older adults should be assessed in future studies.

The finding that postsurgical pain is associated with slowed wound healing provides additional evidence to support aggressive pain management. To the extent that future studies of wound healing identify individual characteristics associated with more or less pain, such as depressive symptoms or the cognitive style of catastrophizing (38), psychological interventions may be an additional valuable treatment component of comprehensive pain management in surgical patients or in illnesses where wounds are associated with pain. The findings of this study suggest that depressive symptoms and pre-existing persistent pain may indicate who is at greater risk for experiencing persistent pain postsurgery and, as a result, who is at greater risk for delayed wound healing. In addition to informing the treatment of surgical patients, knowledge of contributing factors in delayed wound healing may eventually inform interventions in other populations in which slowed healing of chronic wounds is particularly problematic (e.g., in diabetes mellitus) and can lead, in extreme cases, to amputations.

A limitation of this study is the lack of documentation of the amount of pain-related medication used by patients throughout the study period. The extent to which individual differences in 
consumption of opioids and nonsteroidal anti-inflammatory agents may affect wound healing, potentially through effects on the immune system, is an important future direction for studies of pain and wound healing. Although patients in this study did not receive preemptive analgesia (i.e., presurgery epidural analgesia), there is evidence suggesting that preemptive analgesia reduces pain intensity and pain-related disability for several weeks postsurgery $(39,40)$. It is not yet known whether reductions in the experience of postsurgery pain following preemptive analgesia translate into faster wound healing. Additionally, the small sample size of this study, and consequent low statistical power, may have precluded significant results for some analyses (e.g., the association between depressive symptoms on the day of surgery and acute postsurgery pain ratings).

This study examined the influence of depressive symptoms on postsurgery pain and time to healing of wounds; however, there are additional possible psychosocial factors that may interact with pain to affect wound healing or might independently predict wound healing. Prior studies have linked presurgery negative affect, anxiety, and stress with higher postsurgery pain and analgesic use $(28,41)$ and have linked specific coping strategies with postsurgery pain and use of morphine (42). Future studies of the association of postsurgery pain with wound healing should examine the relative contribution of individual differences in pain intensity, emotional distress, social support, and coping on wound healing outcomes. Additionally, we chose to collapse multiple pain ratings into averages for acute and persistent postsurgery pain. An interesting question for a future study is whether the rate of change in postsurgery pain over time is a significant predictor of time to heal wounds.

\section{REFERENCES}

(1) Kendig H, Browning CJ, Young AE: Impacts of illness and disability on the well-being of older people. Disability and Rehabilitation. 2000, 22:15-22.

-(2) Scudds RJ, McD Robertson J: Empirical evidence of the association between the presence of musculoskeletal pain and physical disability in community-dwelling senior citizens. Pain. 1998, 75:229-235.

(3) Morrison RS, Magaziner J, McLaughlin MA, et al.: The impact of post-operative pain on outcomes following hip fracture. Pain. 2003 103:303-311.

(4) Dahl JL, Gordon D, Ward S, et al.: Institutionalizing pain management: The Post-Operative Pain Management Quality Improvement Project. Journal of Pain. 2003, 4:361-371.

(5) Bardiau FM, Taviaux NF, Albert A, Boogaerts JG, Stadler M: An intervention study to enhance postoperative pain management. Anesthesia and Analgesia. 2003, 96:179-185.

(6) Agency for Health Care Policy and Research: Using Clinical Practice Guidelines: Building And Applying a Guideline Based Performance Measurement System-Acute Post-Operative Pain And Urinary Incontinence (Vols. 1 \& 2, ACHPR Pub. No. 95-N013 and 95-N014). Washington, DC: Center for Clinical Quality Evaluation, 1995.

(7) Kehlet H: Acute pain control and accelerated postoperative surgical recovery. Surgery Clinics of North America. 1999, 79:431-443.
(8) Singer AJ, Clark RA: Cutaneous wound healing. New England Journal of Medicine. 1999, 341:738-746.

(9) Marucha PT, Sheridan JF, Padgett DA: Stress and wound healing. In Ader R, Felton DL, Cohen N (eds), Psychoneuroimmunology (3rd Ed.). New York: Academic, 2001, 613-626.

(10) Cuschieri RJ, Morran CG, Howie JC, McArdle CS: Postoperative pain and pulmonary complications: Comparison of three analgesic regimens. British Journal of Surgery. 1985, 72:495-498.

(11) Page GG, Blakely WP, Ben-Eliyahu S: Evidence that postoperative pain is a mediator of the tumor-promoting effects of surgery in rats. Pain. 2001, 90:191-199.

(12) Salomaki TE, Leppaluoto J, Laitinen JO, Vuolteenaho O, Nuutinen LS: Epidural versus intravenous fentanyl for reducing hormonal, metabolic, and physiologic responses after thoracotomy. Anesthesiology. 1993, 79(4):672-679.

(13) Yeager MP, Glass DD, Neff RK, Brinck-Johnsen T: Epidural anesthesia and analgesia in high- risk surgical patients. Anesthesiology. 1987, 66:729-736.

(14) Broadbent E, Petrie KJ, Alley PG, Booth RJ: Psychological stress impairs early wound repair following surgery. Psychosomatic Medicine2. 003, 65:865-869.

(15) Glaser R, Kiecolt-Glaser JK, Marucha PT, et al.: Stress-related changes in proinflammatory cytokine production in wounds. Archives of General Psychiatry. 1999, 56(5):450-456.

(16) Kiecolt-Glaser JK, Marucha PT, Malarkey WB, Mercado AM, Glaser R: Slowing of wound healing by psychological stress. The Lancet. 1995, 346:1194-1196.

(17) Marucha PT, Kiecolt-Glaser JK, Favagehi M: Mucosal wound healing is impaired by examination stress. Psychosomatic Medicine. 1998, 60:362-365.

(18) Padgett DA, Marucha PT, Sheridan JF: Restraint stress slows cutaneous wound healing in mice. Brain Behavior and Immunity. 1998, 12(1):64-73.

(19) Page GG, Ben-Eliyahu S: The immune-suppressive nature of pain. Seminars in Oncology Nursing. 1997, 13:10-15.

(20) Haythornthwaite JA, Sieber WJ, Kerns RD: Depression and the chronic pain experience. Pain. 1991, 46:177-184.

(21) Cole-King A, Harding KG: Psychological factors and delayed healing in chronic wounds. Psychosomatic Medicine. 2001, 63:216-220.

(22) Jensen MP, Turner JA, Romano JM, Fisher LD: Comparative reliability and validity of chronic pain intensity measures. Pain. 1999, 83:157-162.

(23) Ware Jr. JE, Sherbourne CD: The MOS 36-item Short-Form Health Survey (SF-36): I. Conceptual framework and item selection. Medical Care. 1992, 30:473-483.

(24) Beck AT, Beck RW: Screening depressed patients in family practice: A rapid technique. Postgraduate Medicine. 1972, 52:81-85.

(25) Scogin F, Beutler L, Corbishley A, Hamblin D: Reliability and validity of the short form Beck Depression Inventory with older adults. Journal of Clinical Psychology. 1988, 44:853-857.

(26) Kissane DW, Bloch S, Onghena P, et al.: The Melbourne Family Grief Study: II. Psychosocial morbidity and grief in bereaved families. American Journal of Psychiatry. 1996, 153:659-666.

(27) McGuire L, Kiecolt-Glaser JK, Glaser R: Depressive symptoms and lymphocyte proliferation in older adults. Journal of Abnormal Psychology. 2002, 111:192-197. 
(28) Kiecolt-Glaser JK, Page GG, Marucha PT, MacCallum RC, Glaser R: Psychological influences on surgical recovery: Perspectives from psychoneuroimmunology. American Psychologist. 1998, 53:1209-1218.

(29) Kiecolt-Glaser JK, Glaser R: Methodological issues in behavioral immunology research with humans. Brain Behavior and Immunity. 1988, 2:67-78.

(30) Canvas: Version 7.0 [Computer Program]. Deneba Systems, Miami, FL, 1999.

-(31) Schauer PR, Ikramuddin S, Gourash W, Ramanathan R, Luketich J: Outcomes after laparoscopic Roux-en-Y gastric bypass for morbid obesity. Annals of Surgery. 2000, 232:515-529.

(32) Hosmer DW, Lemeshow S: Applied Survival Analysis: Regression Modeling of Time to Event Data. New York: Wiley, 1999.

(33) Stata Statistical Software: Release 8 [Computer Program]. College Station, TX: StatCorp LP, 2003.

(34) Sorensen LT, Karlsmark T, Gottrup F: Abstinence from smoking reduces incisional wound infection: A randomized controlled trial. Annals of Surgery. 2003, 238:1-5.

(35) Linn BS, Linn MW, Klimas NG: Effects of psychophysical stress on surgical outcome. Psychosomatic Medicine. 1988, 50:230-244.

(36) Rojas IG, Padgett DA, Sheridan JF, Marucha PT: Stress-induced susceptibility to bacterial infection during cutaneous wound healing. Brain Behavior and Immunity. 2002, 16:74-84.
(37) Kiecolt-Glaser JK, McGuire L, Robles TF, Glaser R: Emotions, morbidity, and mortality: New perspectives from psychoneuroimmunology. Annual Review of Psychology. 2002, 53:83-107.

(38) Sullivan MJ, Thorn B, Haythornthwaite JA, et al.: Theoretical perspectives on the relation between catastrophizing and pain Clinical Journal of Pain. 2001, 17(1):52-64.

(39) Katz J, Cohen L: Preventive analgesia is associated with reduced pain disability 3 weeks but not 6 months after major gynecologic surgery by laparotomy. Anesthesiology. 2004, 101:169-174.

(40) Katz J, Cohen L, Schmid R, Chan VW, Wowk A: Postoperative morphine use and hyperalgesia are reduced by preoperative but not intraoperative epidural analgesia: Implications for preemptive analgesia and the prevention of central sensitization. Anesthesiology. 2003, 98:1449-1460.

(41) Ozalp G, Sarioglu R, Tuncel G, Aslan K, Kadiogullari N: Preoperative emotional states in patients with breast cancer and postoperative pain. Acta Anaesthesiologica Scandinavica. 2003, 47:26-29.

(42) Cohen L, Fouladi RT, Katz J: Preoperative coping strategies and distress predict postoperative pain and morphine consumption in women undergoing abdominal gynecologic surgery. Journal of Psychosomatic Research. 2005, 58:201-209. 